\title{
Um Algoritmo Paralelo Eficiente para Cálculo de Centralidade em Grafos
}

\author{
Leonardo Carlos da Cruz \\ Departamento de Computação \\ CEFET-MG \\ Belo Horizonte, Brasil \\ Email: leonardocruz@ufmg.br
}

\author{
Cristina Duarte Murta \\ Departamento de Computação \\ CEFET-MG \\ Belo Horizonte, Brasil \\ Email: cristina@decom.cefetmg.br
}

\begin{abstract}
Resumo-Estudos em áreas tão diversas quanto sistemas de comunicação, Internet e Web, computação móvel, sistemas biológicos, redes sociais e redes de transporte, dentre outras, apresentam em comum o fato de que todos esses sistemas podem ser modelados por grafos. A quantidade de elementos participantes nessas redes complexas tem alcançado escalas cada vez maiores, o que requer o uso de processamento paralelo para a análise dos grafos. Neste artigo apresentamos um novo algoritmo paralelo para o cálculo exato de centralidades em grafos grandes, usando o paradigma de programação MapReduce/Hadoop. O objetivo é computar as distâncias entre os vértices e extrair medidas de centralidades decorrentes dessas distâncias, fazendo uso eficiente dos recursos computacionais. Para avaliar o algoritmo proposto foram processados diversos grafos e os resultados comparados com algoritmos sequenciais e paralelos. Os experimentos mostraram que o algoritmo proposto faz uso eficiente da memória e do espaço em disco, comparado à outras implementações.
\end{abstract}

Palavras-chave: processamento paralelo, grafos, redes complexas.

\section{INTRODUÇÃO}

A crescente facilidade de aquisição de dados associada à ampla disponibilidade de dispositivos de armazenamento possibilitou o surgimento de grandes massas de dados em diversos contextos. Assim, em várias áreas do conhecimento, da coleta de amostras de moléculas de DNA, capazes de gerar milhões de fragmentos advindos de uma simples bactéria, até a aquisição de imagens e medidas de radiações capturadas por telescópios que varrem o universo [1], há cada vez mais dados para processar e armazenar. Um modelo comumente usado para representar tais conjuntos de dados são os grafos. Portanto, explorar modelos baseados em grafos é uma importante tarefa para auxiliar a descoberta científica. Contudo, desenvolver técnicas eficientes capazes de extrair propriedades em grafos é uma tarefa não trivial, e o desafio torna-se ainda maior quando estamos trabalhando com grafos grandes, em que o número de arestas e vértices alcançam escalas de milhões ou maiores.

No contexto do processamento paralelo de grandes quantidades de dados, o modelo de programação paralela MapReduce foi adotado por empresas de tecnologia da informação e no meio acadêmico. Um dos fatores que permitiram seu uso foi o desenvolvimento da plataforma Hadoop, uma implementação open source do modelo, que computa com sucesso dados na escala de petabytes. Assim, tornou-se desejável aplicar esse modelo ao processamento de grafos grandes.

Nesse contexto, apresentamos o AHEAD (Advanced Hadoop Exact Algorithm for Distances), um algoritmo paralelo para processamento de grafos grandes, implementado em MapReduce/Hadoop. Nosso objetivo é investigar a aplicabilidade do modelo MapReduce ao processamento de grafos, particularmente ao processamento das distâncias entre os vértices, bem como a escalabilidade desse processamento em grafos com quantidades de vértices e arestas em várias escalas, e também o uso eficiente dos recursos computacionais no procedimento.

A computação de medidas de centralidade em grafos apresenta barreiras no que diz respeito à complexidade de sua computação [2]. Essas medidas, que incluem o diâmetro e o raio do grafo, tornam-se difíceis, senão proibitivas, de serem calculadas em grafos de escala muito grande [3]. Assim, a questão a ser avaliada é que tamanhos de grafos podemos analisar, por meio de algoritmos paralelos, para obter suas medidas exatas de centralidade. Para grafos maiores, podemos ter apenas um cálculo aproximado destas medidas [3], [4].

Esse trabalho dá continuidade ao trabalho desenvolvido em [5], em que foi proposto o algoritmo HEDA (Hadoop based Exact Diameter Algorithm), que encontra medidas exatas de centralidade para grafos de tamanhos moderados. No entanto, o algoritmo HEDA apresenta limitações quanto ao uso do espaço de armazenamento de dados [6]. Essas limitações têm relação com o uso que o algoritmo faz da memória principal e o modelo de armazenamento de dados do Hadoop no sistema de arquivos. Para contornar essas limitações, o AHEAD aplica as técnicas propostas no trabalho [7], o que permite avanços na obtenção de medidas exatas de diâmetro e raio em grafos de escalas maiores. Os resultados apresentados nesse artigo mostram melhorias significativas quanto ao uso da memória principal e espaço em disco em relação a outras implementações.

\section{TRABALHOS RELACIONADOS}

\section{A. Modelo MapReduce}

O modelo de programação MapReduce tem por objetivo descrever uma maneira de processar grandes quantidades de dados de forma paralela [8]. Nesse modelo considera-se que o volume de dados a ser processado não é compatível com a capacidade de armazenamento de um único nó computacional. Portanto, em seu projeto, assume-se o uso 


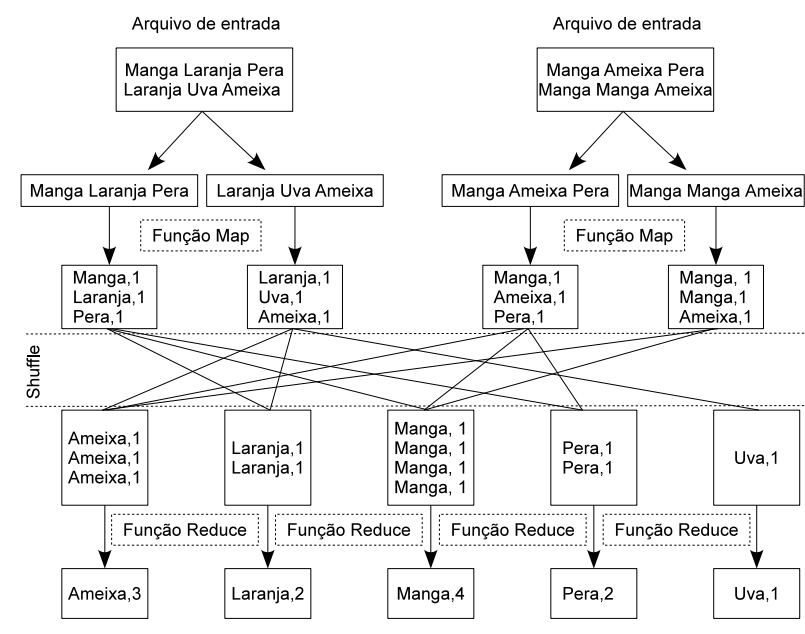

Figura 1. O modelo MapReduce para um contador de palavras.

de vários desses nós. Assim, o modelo inclui uma dinâmica de conexões entre os recursos computacionais, cujo objetivo é distribuir de forma coerente os dados e o processamento. Adicionalmente, o MapReduce foi projetado para ser usado em aglomerados de máquinas comuns [8]. Essa condição permite flexibilidade na redução de custos com as mesmas, o que é especialmente interessante quando a expectativa é usar milhares de nós computacionais. Diante dessa perspectiva, o modelo também prevê a manutenção da confiabilidade no processamento, mesmo que a probabilidade de falhas aumente não apenas devido a uma menor robustez das máquinas, mas também devido ao número de máquinas presentes no sistema.

Do ponto de vista de programação paralela, o MapReduce especifica apenas duas funções que devem ser escritas pelo programador: a função Map e a função $R e-$ duce. A programação necessária para a sincronização dos processamentos, distribuição dos dados e o uso adequado dos recursos computacionais está presente em camadas do modelo que não são visíveis ao programador, sendo acessíveis apenas pela configuração de parâmetros do modelo MapReduce/Hadoop. Esse paradigma de programação, portanto, procura reduzir a complexidade do desenvolvimento de programas paralelos para grandes volumes de dados, tipicamente na escala de petabytes, provendo confiabilidade no processamento através do gerenciamento de falhas nas máquinas constituintes do sistema distribuído [9].

Um esquema de funcionamento do modelo MapReduce é apresentado na Figura 1. A ideia básica do modelo é processar paralelamente vários arquivos de entrada usando uma função Map e uma função Reduce. No exemplo da Figura 1, o objetivo é contar as palavras contidas nos documentos armazenados no sistema de arquivo distribuído HDFS (Hadoop Distributed File System). Inicialmente, a função Map recebe como entrada um par arquivo/conteúdo, processando uma linha do arquivo por vez. A saída do processamento é o par constituído por uma palavra, tomada como chave, e pelo valor númerico 1. Cada par chave/valor é armazenado em partições nos discos locais da máquina que executa a função Map, onde a partição de destino é determinada por uma função particionadora. Após todos os arquivos de entrada terem sido processados paralelamente, as saídas das funções Map são redistribuídas pelo sistema, em um procedimento chamado shuffle [10].

Nesse processo, as saídas das funções Map com palavras ou chaves idênticas são agrupadas na mesma partição, sendo estas armazenadas no sistema de arquivo local das máquinas que irão executar a função Reduce. Ao fim da redistribuição, a fase seguinte é realizada pela execução da função Reduce, aplicada paralelamente em cada partição. À medida que a função é aplicada, a saída é gravada no HDFS. No exemplo da Figura 1, a função Reduce soma todos os valores associados a mesma chave, resultando na contagem das palavras contidas nos arquivos de entrada.

\section{B. Cálculo de centralidade}

Para este trabalho consideramos grafos direcionados $G=(V, A)$, sendo $V$ o conjunto de vértices e $A$ o conjunto de arestas direcionadas, onde as arestas representam alguma relação existente entre dois vértices. Grafos não direcionados podem ser representados substituindo-se as arestas não direcionadas por duas arestas direcionadas em sentidos opostos. Os grafos aqui tratados são simples, ou seja, sem arestas múltiplas, sem laços e sem pesos nas arestas.

Em diversos modelos matemáticos que utilizam grafos, a noção de centro de uma rede é bastante explorada, pois muitas vezes deseja-se determinar a localização de elementos no grafo que atendam algum critério de satisfação. Por exemplo, pode-se querer determinar um ponto na rede em que o tempo de acesso aos demais pontos seja o menor possível. Em outros casos, deseja-se que esse tempo tenha um limite superior, ou seja, deseja-se que o tempo de resposta entre tal ponto e os demais seja fixo.

Nesse trabalho em particular, estamos interessados no cálculo do diâmetro e raio de um grafo, feito a partir do cálculo de excentricidades que, por sua vez, são calculadas a partir das distâncias entre os vértices do grafo. A distância $d(s, t)$ de um vértice $s$ até um vértice $t$ é o comprimento do menor caminho entre $s$ e $t$. A excentricidade de um vértice é a maior distância entre esse vértice e os demais vértices alcançáveis da rede. Assim, denotamos a excentricidade de um vértice por $e(v)=\max _{t \in V}\{d(v, t)\}$.

A maior das excentricidades entre os vértices é definida como o diâmetro do grafo, ou seja, é a maior distância entre dois vértices presentes no grafo. Matematicamente, expressamos o diâmetro de um grafo $G=(V, A)$ por $D(G)=\max _{v \in V}\{e(v)\}$. O raio do grafo, denotado por $R(G)=\min _{v \in V}\{e(v)\}$, é a menor excentricidade entre os vértices do grafo. A análise da excentricidade revela que vértices com valores de excentricidade baixos são centrais ao grafo, enquanto valores de excentricidade elevados indicam os vértices periféricos ao grafo. Nota-se que essa medida de centralidade tem custo computacional elevado, pois a quantidade de menores caminhos é estimada supe- 
riormente pelo cálculo $\left(\begin{array}{c}|V| \\ 2\end{array}\right)=\frac{|V|^{2}-|V|}{2}$ para grafos de $|V|$ vértices. Além disso, deve-se levar em consideração o custo computacional necessário para encontrar um único menor caminho.

\section{Processamento paralelo de grafos usando MapReduce}

Antes de processar dados no modelo MapReduce é necessário fazer uma análise da adequabilidade da aplicação alvo a esse paradigma de programação. Alguns algoritmos mais complexos não são de fácil adaptação ao modelo, enquanto outros são inerentemente paralelizáveis, com o melhor desempenho do MapReduce sendo mais evidente neste último grupo [11]. Para o caso de processamento de grafos, é possível implementar operações em grafos de forma simples e eficiente, desde que essas operações possam ser caracterizadas por permitir processamento local [12].

No trabalho realizado em [7], os autores mostram um conjunto de boas práticas para o desenvolvimento de projeto de algoritmos para grafos usando o modelo MapReduce. Essas práticas são compostas por três técnicas, chamadas In-Mapper Combiner, Schimmy e Range Partitioning, todas inspiradas na dinâmica de funcionamento do modelo. A técnica In-Mapper Combiner consiste em préprocessar a saída da fase Map, reduzindo a quantidade de dados transferidos pela fase Shuffle e diminuindo o trabalho realizado pela fase Reduce. Em outras palavras, a técnica objetiva reduzir o processamento na fase Reduce com a diminuição do tamanho da saída da fase Map. Quando se usa técnica Schimmy, a intenção é transmitir da maneira mais eficiente possível, entre uma fase e outra, informações sobre a estrutura do grafo. Por fim, a técnica Range Partitioning consiste em projetar uma função de partição que aproveita informações sobre a topologia do grafo. Por exemplo, é mais interessante criar uma função particionadora que aloca vértices vizinhos na mesma partição de saída da fase Map, pois assim qualquer processamento que envolva ambos é feito localmente, ou seja, na memória do mesmo nó computacional. Combinando essas três práticas de projeto, os autores conseguiram ganhos de desempenho de até $70 \%$ em relação a implementações do algoritmo PageRank [13].

\section{Algoritmo HADI}

O algoritmo HADI (HAdoop DIameter and radii estimator) [4] é um algoritmo implementado em MapReduce/Hadoop para estimar o diâmetro de grafos com tamanhos na ordem de petabytes. Para fazer o cálculo aproximado do diâmetro, o algoritmo faz uso do conceito de diâmetro efetivo, cuja definição é o comprimento mínimo em que $90 \%$ dos pares de vértices conectados no grafo são alcançáveis entre si. A principal operação do algoritmo é estimar, paralelamente, a contagem do número de pares conectados que se alcançam dentro de um determinado comprimento de caminho, usando para isso o algoritmo de Flajolet-Martin [14]. Ao estimar a contagem de pares conectados, é possível fazer o cálculo aproximado do diâmetro do grafo. $\mathrm{O}$ algoritmo alcança bons resultados

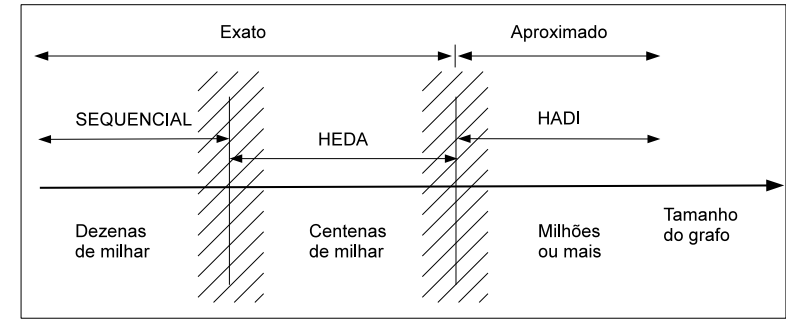

Figura 2. Faixas de atuação dos algoritmos HEDA, HADI e sequencial no cálculo de diâmetro em grafos de diversos tamanhos.

em termos de escalabilidade com o número de máquinas e em termos de tempo de execução com o número de vértices. No entanto, o diâmetro estimado pode diferir significativamente de seu valor real [5].

\section{E. Algoritmo HEDA}

Também desenvolvido para a plataforma MapReduce/ Hadoop, o algoritmo HEDA (Hadoop-based Exact Diameter Algorithm) [5] é um algoritmo baseado na expansão de fronteiras para descoberta de vértices vizinhos (busca em largura). O algoritmo faz a expansão paralelamente, calculando a distância entre um nó de origem e todos os outros de forma simultânea e exata, na medida que avança na descoberta de novos vértices.

A Figura 2 exibe as escalas de tamanhos de grafos em que os algoritmos HEDA, HADI e sequencial atuam, em seus cálculos exatos e aproximados do diâmetro do grafo. A região hachurada indica que os limites de atuação de cada algoritmo apresentam alguma flexibilidade, dependendo do grafo e dos recursos computacionais utilizados no processamento. Há um espaço para atuação do algoritmo HEDA que é explorado através do paralelismo, a fim de obter resultados exatos para grafos que são impossíveis de serem processados por soluções sequenciais.

Tendo em vista a impossibilidade atual de se calcular o valor exato de métricas de centralidade em grafos muito grandes, a pergunta que surge é: para que tamanhos de grafos (no de vértices e arestas) é possível fazer o cálculo exato do raio e do diâmetro? Essa pergunta se faz necessária pois, para aqueles interessados no cálculo do diâmetro de grafos grandes, cabe escolher qual resultado exato ou aproximado - melhor atende as suas necessidades.

\section{DESCRIÇÃO DO ALGORITMO AHEAD}

\section{A. Visão Geral}

No caso do processamento paralelo de grafos usando o modelo MapReduce, a divisão dos dados de entrada significa a divisão da estrutura do grafo no sistema distribuído. Portanto, dado um certo número de mappers, que são máquinas virtuais Java instanciadas pela plataforma Hadoop, a estrutura do grafo deve ser distribuída entre os mesmos na forma de subconjuntos excludentes. Contudo, o mapper que executa a função Map em um subconjunto não compartilha os dados na sua memória com os demais mappers do sistema distribuído. Assim, para calcular e atualizar as distâncias entre vértices de partições ou máquinas diferentes, é necessário transmitir os resultados locais entre as partições do grafo. Para tanto os mappers 
armazenam temporariamente os resultados intermediários no sistema de arquivo local para repassá-los às iterações seguintes do processamento.

A recepção e a síntese dos resultados parciais é feita pelos reducers, que assim como os mappers, são máquinas virtuais Java instanciadas e gerenciadas pela plataforma Hadoop. A síntese diz respeito a necessidade de, dentre as informações recebidas pelos reducers, selecionar aquelas que atendam algum critério de satisfação relacionado ao problema proposto. No caso do algoritmo AHEAD, o critério definido é a menor distância calculada até o momento entre dois vértices do grafo. Além disso, o reducer deverá acessar as partições as quais tais vértices pertencem, com o propósito de realizar a devida atualização da informação sobre a distância entre eles.

\section{B. Funcionamento do Algoritmo}

$\mathrm{O}$ algoritmo AHEAD utiliza as ideias expostas no trabalho realizado em [7], com a implementação das técnicas In-Mapper Combiner e Schimmy. A técnica Range Partitioning não é implementada por ser efetiva apenas para grafos que representam domínios da Web. Porém, a ação de particionar o grafo é de fato implementada no AHEAD, com o uso da função Default Partitioner do Hadoop. Essa função particiona os dados de entrada baseandose em uma função hash, o que proporciona partições com tamanhos homogêneos. Partições de mesmo tamanho permitem que o processamento das mesmas termine em tempos aproximadamente iguais, evitando assim a contenção na execução paralela devido a um balanceamento ruim na distribuição dos dados. Referimemos a esse tipo de particionamento como Hash Partitioning.

O objetivo ao implementar as técnicas mencionadas foi otimizar o uso do espaço em disco e memória. Tal otimização possibilita o avanço nas escalas de tamanhos dos grafos que podem ser processados de forma exata, de acordo com o sentido exibido na Figura 2. O pseudocódigo do algoritmo AHEAD, mostrado na Figura 3, é composto pelas três fases listadas a seguir:

1. Linhas (1-6): Criação paralela no HDFS da matriz de distâncias do grafo. A matriz é particionada em $\mathrm{R}$ partições, onde $\mathrm{R}$ é o número de reducers;

2. Linhas (7-13): Cálculo paralelo das distâncias entre todos os vértices e atualização paralela no HDFS das partições matriciais representativas do grafo;

3. Linhas (14-24): Cálculo paralelo da excentricidade de todos os vértices. As excentricidades são usadas para o cálculo de raio e diâmetro do grafo.

A representação matricial do grafo informa que a posição de linha $i$ e coluna $j$ da matriz é ocupada pelo valor da distância entre o vértice de destino $i$ e o vértice de origem $j$. A matriz é criada na fase 1 a partir da leitura de todos os registros da lista de adjacências do grafo. A lista de adjacências contém em cada registro um vértice do grafo e a sua lista de vizinhos. Na linha (4), mappers enviam em paralelo os registros da lista de adjacências para uma determinada partição $G_{i}$. Na linha
1: $G \leftarrow$ lista_de_adjacências;

2: $R \leftarrow$ número_de_reducers;

3: for $i=1$ to $R$ do in parallel

4: $\quad G_{i} \leftarrow$ CriaParticaoDeMatriz $(G)$;

5: $\quad$ HDFS $\leftarrow G_{i}$;

6: end for

7: while (condição de parada $=$ false) do

for all $G_{i} \in G=\left\{G_{1} \cup G_{2} \cup \ldots \cup G_{R}\right\}$ do in parallel CalculaMenoresCaminhos $\left(G_{i}\right)$; AtualizaDistancias $\left(G_{i}\right)$;

end for

Atualiza(condição de parada);

end while

for all $G_{i} \in G=\left\{G_{1} \cup G_{2} \cup \ldots \cup G_{R}\right\}$ do in parallel

for all vértice $v \in G_{i}$ do

CalculaExcentricidade $(v)$

$D_{i} \leftarrow$ MaiorExcentricidade();

$R_{i} \leftarrow$ Menor Excentricidade () ;

end for

end for

repeat in parallel

Diâmetro $\leftarrow$ RemoveMenor $\left(\left\{D_{1}, D_{2}, \ldots, D_{R}\right\}\right)$; Raio $\leftarrow$ RemoveMaior $\left(\left\{R_{1}, R_{2}, \ldots, R_{R}\right\}\right)$;

until $\left\{D_{1}, D_{2}, \ldots, D_{R}\right\}=\emptyset$ and $\left\{R_{1}, R_{2}, \ldots, R_{R}\right\}=\emptyset$

Figura 3. Programa Principal com as três fases do algoritmo AHEAD.

(5), reducers recepcionam e fazem a expansão dos registros com as colunas correspondentes às distâncias para os outros vértices do grafo. A partição a qual o registro é enviado e posteriormente estendido é determinada pela função Hash Partitioning. Ao final dessa fase, existirão $R$ arquivos no HDFS, cada um contendo uma parte da matriz de distâncias do grafo. Essa é a única fase em que a estrutura do grafo é transmitida via processo shuffle, o que coloca o AHEAD de acordo com o padrão de projeto Schimmy proposto em [7].

$\mathrm{Na}$ linha (9) da Figura 3, mappers executam em paralelo as operações do cálculo de distâncias entre os vértices do grafo, de acordo com as informações disponíveis em uma partição $G_{i}$. A operação de cálculo da distância é baseada no algoritmo de busca em largura, onde a distância acumulada de um vértice em relação a uma origem serve de referência para o cálculo da distância de seus vizinhos em relação à mesma origem. Portanto, para calcular a distância de um vértice vizinho, basta adicionar um à distância acumulada. A função Map que calcula os caminhos entre as diversas origens e destinos foi implementada de forma que somente os menores valores são gravados localmente antes de serem enviados aos reducers. Além disso, quando há caminhos diferentes entre origem e destino com valores de distâncias iguais, apenas um desses valores fica disponível, evitando redundância de informação no disco local. Essas duas técnicas de implementação da função Map contemplam o padrão de projeto In-Mapper Combiner [7].

Na linha (10), os reducers acessam a partição do grafo, isto é, o arquivo do grafo que receberá as atualizações das distâncias. Cada reducer é responsável por uma partição. A atualização das $R$ partições ocorre simultaneamente, isto é, os reducers trabalham em paralelo assim como os mappers o fazem durante o cálculo das distâncias. Sempre que a distância de um vértice é atualizada, o cálculo da distância 
de seus vértices vizinhos em relação a origem considerada é disparada na próxima iteração. A condição de parada na linha (12) é baseada nesse fato, pois o reducer que atualiza alguma partição também incrementa um contador global de atualizações. Uma iteração completa do laço na linha (7) corresponde a um ciclo map-shuffle-reduce ou um $J o b$. Quando não houver atualizações durante um Job, a condição na linha (12) muda, encerrando o laço. Note que no laço somente as distâncias calculadas são transferidas entre mappers e reducers. Não há transferência da estrutura do grafo. Tal fato economiza o espaço usado para armazenamento local dos resultados parciais, como também economiza recursos de rede usados para a transferência dos mesmos entre as instâncias de máquinas virtuais Java.

\section{Número de Mappers e Reducers}

A quantidade de mappers instanciados pela plataforma Hadoop depende do tamanho da entrada de dados. O número de mappers $M$ é o resultado da divisão:

$$
M=\frac{T_{e}}{B_{\mathrm{hdfs}}},
$$

sendo $T_{e}$ a quantidade de bytes na entrada da fase Map e $B_{\text {hdfs }}$ um parâmetro configurável do Hadoop que especifica o tamanho em bytes do bloco de dados usado pelo HDFS. Um arquivo armazenado no HDFS é constituído por um ou mais blocos dependendo do valor de $B_{\text {hdfs }}$ e, de acordo com a equação (1), a plataforma Hadoop aloca por default um mapper para cada bloco de dados. O número $R$ de reducers instanciados pelo Hadoop é especificado via parâmetro no AHEAD, sendo que o valor usado nesse trabalho é sempre igual a quantidade de cores disponíveis no sistema. Assim, considerando $c$ o número de cores no cluster e $k=\frac{M}{R}$ a relação entre o número de mappers e reducers desejada, ao dividirmos os dois lados da equação (1) por $R$ obtemos:

$$
B_{\mathrm{hdfs}}=\frac{T_{e}}{k \cdot c}
$$

Se $k=1$ e $T_{e}$ é conhecido, o valor calculado de $B_{\text {hdfs }}$ é tal que o número de mappers ou reducers alocados pela plataforma Hadoop iguala ao número de cores disponíveis no sistema. A implementação modular do AHEAD permite que a sua primeira fase seja executada isoladamente, o que viabiliza a verificação prévia do valor de $T_{e}$. Assim é possível estimar o ajuste de $B_{\text {hdfs }}$ de forma que as relações cores/mappers e cores/reducers sejam 1/1.

\section{Resultados}

\section{A. Planejamento dos experimentos}

O algoritmo AHEAD foi avaliado mediante o processamento de grafos reais e sintéticos. Os grafos reais foram obtidos na base de dados Stanford Large Network Dataset Collection ${ }^{1}$ mantida pelo grupo de pesquisa Stanford Network Analysis Project. Os grafos reais utilizados são o da rede social Epinions, com 75.879 vértices e

${ }^{1}$ http://snap.stanford.edu/data/index.html
508.837 arestas, o grafo da rede de comunicação por correio eletrônico de funcionários da empresa Enron, com 36.692 vértices e 367.662 arestas, e a rede de colaboração científica entre co-autores de artigos da categoria Astro Physics da revista eletrônica arXiv, com 18.772 vértices e 396.160 arestas.

Além desse conjunto, foram criados grafos sintéticos com número de vértices fixo em 50.000 (50K) e número de arestas variando entre $150 \mathrm{~K}$ e $1.600 \mathrm{~K}$, com o objetivo de avaliar o escalonamento do AHEAD quanto à variação do número de arestas. Também foram gerados grafos sintéticos com número de vértices variando entre $25 \mathrm{~K}$ e $200 \mathrm{~K}$ e arestas variando entre $75 \mathrm{~K}$ e $600 \mathrm{~K}$. Para a geração de grafos sintéticos foi utilizada a biblioteca NetworkX [15], que também foi a fonte de um dos dois algoritmos sequenciais utilizados. O segundo algoritmo sequencial testado no trabalho é citado em [16]. Os resultados de ambos são comparados com o AHEAD para verificação da correção dos resultados (raio e diâmetro dos grafos). Uma comparação entre HEDA e AHEAD é feita com o objetivo de verificar o uso de espaço em disco e memória. Os valores apresentados em gráficos e tabelas são a média dos resultados de três processamentos. Observamos que a variação dos valores em torno da média é pequena. Os dados podem ser encontrados em [17].

Os testes foram realizados em um cluster com 6 nós de processamento interligados via switch de $1 \mathrm{~Gb} / \mathrm{s}$, onde cada nós possui dois processadores Intel Xeon X5660 a 2,80 GHz, 98 GB de RAM e $300 \mathrm{~GB}$ de disco. Ao todo são 72 cores físicos ou 144 cores virtuais (hyperthreading) presentes no cluster. A versão Hadoop instalada é 1.1.2 e o sistema operacional é o Linux Gentoo Kernel 3.6.11. Somente durante a comparação entre os algoritmos HEDA e AHEAD, na Seção IV-F, a capacidade dos discos locais foram trocadas para o valor máximo disponível, que é de 2TB por máquina.

\section{B. Correção do algoritmo}

O AHEAD gerou resultados de raio e diâmetro iguais aos dos algoritmos sequenciais e do HEDA, para vários grafos testados. Por exemplo, para o grafo sintético com $50 \mathrm{~K}$ vértices e $200 \mathrm{~K}$ arestas, todos os algoritmos testados apresentaram os mesmos resultados, a saber, Diâmetro $=15$ e Raio $=11$. A Figura 4 mostra os tempos de execução dos algoritmos sequenciais (em uma máquina), e dos algoritmos HEDA e AHEAD, quando varia-se o número de máquinas. Os resultados exibidos na Figura 4 indicam que o modelo MapReduce/Hadoop apresenta um overhead significativo, e seu uso só se justifica para quantidades de dados que não podem ser executadas em uma única máquina.

\section{Escalabilidade}

1) Variação de arestas: O comportamento do algoritmo AHEAD frente à variação no número de arestas é exibido nas Figuras 5 e 6 . Nesse experimento, o grafo tem $50 \mathrm{~K}$ vértices e o número de arestas varia entre $200 \mathrm{~K}$ e $1.600 \mathrm{~K}$. $\mathrm{O}$ aumento do número de arestas representa o aumento 


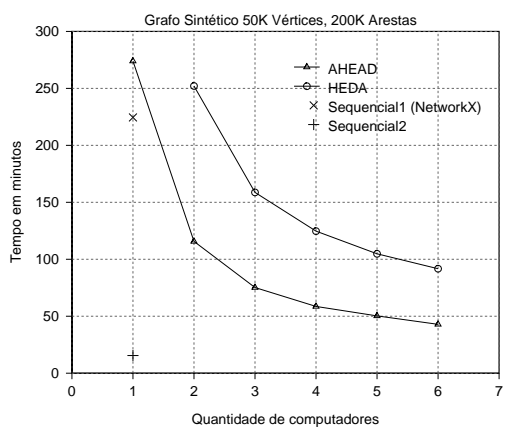

Figura 4. Comparação entre AHEAD, HEDA e algoritmos sequenciais.

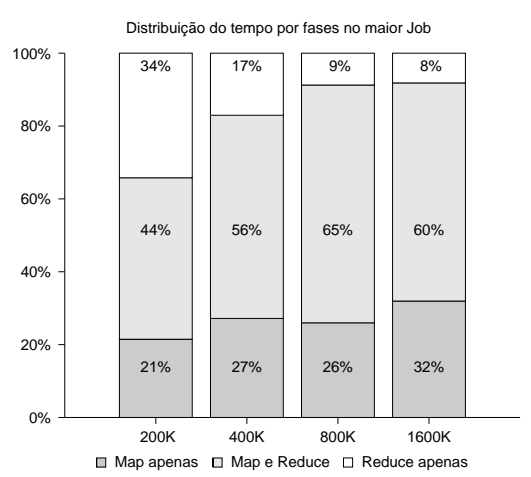

Figura 5. Tempo gasto em cada fase com o aumento do número de arestas. Os dados são coletados do job de maior duração.

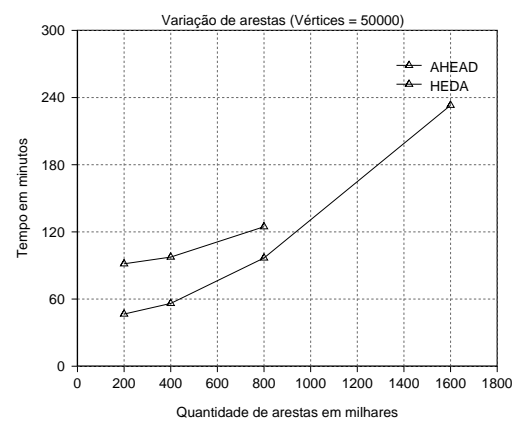

Figura 6. Variação no número de arestas em grafos de $50 \mathrm{~K}$ vértices. no número de relações entre uma quantidade fixa de entidades. A Figura 6 mostra que a taxa de crescimento do tempo de execução é lenta. No intervalo testado, o tempo de execução do AHEAD foi multiplicado por cerca de quatro enquanto o número de arestas foi multiplicado por oito. A Figura 5 mostra que, a partir de $400 \mathrm{~K}$ arestas, a fase map, que faz a busca em largura no grafo, domina o tempo de processamento gasto no Job com maior tempo de duração. Para esse gráfico, o tempo de processamento da fase reduce inclui o tempo gasto no processo de transmissão de dados (shuffle). Nas Figuras 4 e 6, respectivamente, o algoritmo HEDA não processa o grafo com $200 \mathrm{~K}$ arestas em uma máquina e $1.600 \mathrm{~K}$ arestas em seis máquinas. Nos dois casos, o sistema acusa falta de espaço em disco no cluster durante o processamento.

2) Variação de vértices e arestas: A Tabela I mostra os valores de diâmetro e tempo de processamento de grafos sintéticos quando altera-se o número de arestas e vértices. $\mathrm{O}$ aumento do número de vértices e arestas nesse caso representa o aumento do número de entidades e do número de relações entre elas. Na prática, a inserção de vértices só tem efeito no cálculo das distâncias se os mesmos tiverem grau maior ou igual a um. Os grafos exibidos na Tabela I são conectados, o que garante a existência de no mínimo uma nova aresta a cada novo vértice. Nessa tabela, percebe-se que a variação do tempo de execução com a variação do tamanho do grafo é maior se comparada à variação mostrada na Figura 6. Ao aumentar o valor de vértices e arestas $(V+A)$, mantendo a relação arestas/vértices relativamente baixa $(A / V=3)$, estamos aumentando a quantidade de vértices distantes entre si, o que dificulta a exploração do algoritmo sobre o grafo. Tal fato afeta o comportamento do algoritmo, pois quando tem-se uma relação arestas/vértice baixa, haverá também menos opções de caminhos entre os vértices. $\mathrm{O}$ número reduzido de caminhos faz o algoritmo ter que encontrar vértices mais isolados ou distantes entre si, resultando nos diâmetros cada vez maiores mostrados na Tabela I.

\section{Speedup e Eficiência}

Para os testes de desempenho foram usados os grafos reais descritos na Seção IV-A, juntamente com um grafo sintético contendo $50 \mathrm{~K}$ vértices e $200 \mathrm{~K}$ arestas. A Figura 7 mostra os resultados de tempo de execução, speedup e eficiência frente à variação do número de máquinas do cluster. Nota-se no gráfico da Figura $7 \mathrm{~b}$ que, para grafos maiores, o AHEAD apresenta Speedup superlinear. Isso pode ser explicado pelo fato de que, ao acrescentar máquinas no cluster, a quantidade de dados que um nó computacional deve processar é menor, permitindo que os dados em processamento possam ser melhor ajustados nas memórias cache de cada processador. Assim, as operações de memória serão executadas em cada nó computacional de maneira mais eficiente. Esse efeito também aparece no gráfico da Figura 7c, onde há valores de eficiência maiores do que 1. Para grafos menores, entendemos que tal efeito é sobreposto pelo overhead que o sistema apresenta, que é devido a vários fatores relacionados ao processamento distribuído dos mappers e reducers, como o instanciamento das máquinas virtuais, o gerenciamento de falhas e a comunicação de dados via rede ethernet na fase shuffle.

\section{E. Variação do tamanho dos blocos no HDFS}

De acordo com a Equação 2 da Seção III-C, a relação entre o número de mappers e reducers, $k$, pode ser ajustada pela seleção adequada do valor de $B_{\text {hdfs, }}$ que é o tamanho do bloco usado no HDFS. A Figura 8 mostra a distribuição de mappers e reducers no cluster para $k=1$ e $k=2$, em uma máquina com 23 cores. Os gráficos são gerados a partir dos dados da sequência mapshuffle-reduce (Job) de maior tempo de duração, durante o processamento do grafo Epinions. Uma barra horizontal no gráfico tem o comprimento relativo à duração de uma tarefa, considerando as datas de início e de fim no formato epoch do Unix. Na Figura 8a, notamos a predominância Tabela I

EFEITO DA VARIAÇ̃̃o DO NÚMERO DE VÉRTICES E ARESTAS EM GRAFOS SINTÉTICOS.

\begin{tabular}{ccccc}
\hline Vértices / Arestas & Diâmetro & $\begin{array}{c}\text { Tempo médio } \\
\text { (minutos) }\end{array}$ & $\begin{array}{c}\text { Variação } \\
\text { tamanho }\end{array}$ & $\begin{array}{c}\text { Variação } \\
\text { tempo }\end{array}$ \\
\hline $25.000 / 75.000$ & 21 & 19,5 & - & - \\
$50.000 / 150.000$ & 23 & 47,1 & $2 \mathrm{x}$ & $2,4 \mathrm{x}$ \\
$100.000 / 300.000$ & 24 & 163,3 & $4 \mathrm{x}$ & $8,4 \mathrm{x}$ \\
$200.000 / 600.000$ & 26 & 1129,8 & $8 \mathrm{x}$ & $57,9 \mathrm{x}$ \\
\hline
\end{tabular}




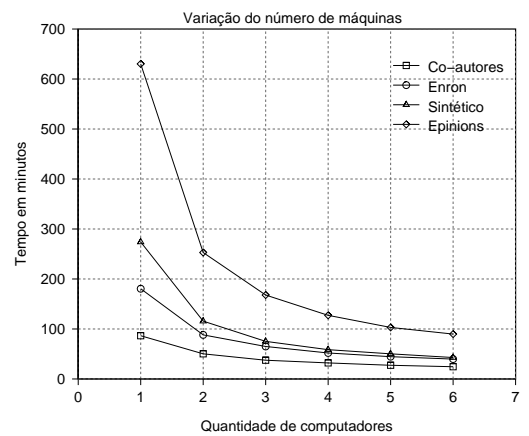

(a) Tempo total.

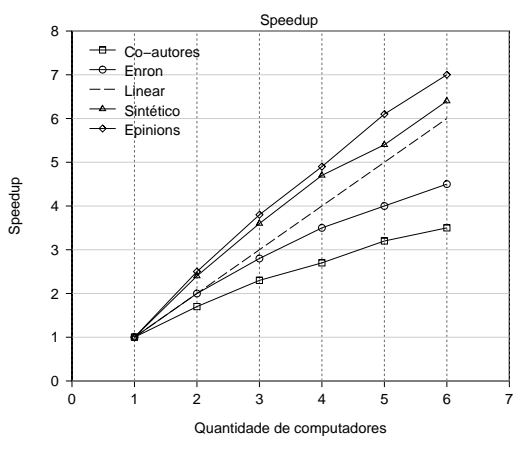

(b) Speedup.

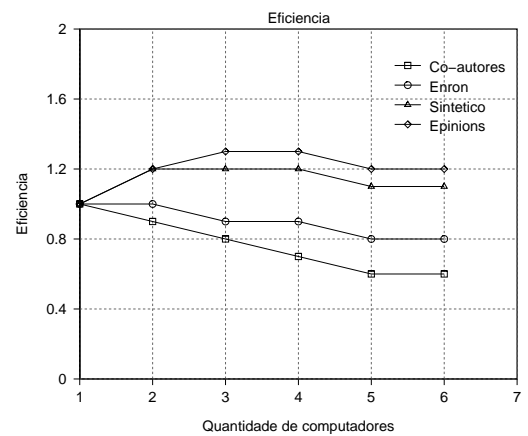

(c) Eficiência.

Figura 7. Teste de desempenho.

do paralelismo entre tarefas map e entre tarefas reduce de forma isolada, isto é, há pouca concorrência entre mappers e reducers. A Figura $8 \mathrm{~b}$ mostra predominância na concorrência entre tarefas de natureza diferente. Notamos também que, para $k=2$, o instanciamento de tarefas map ocorre em grupos de 23 mappers, que é o número de cores. No eixo Y, o último grupo de mappers é constitíudo pelas tarefas 47 a 69 . Este grupo se deve ao fato de que o valor de $T_{e}$ foi acima do valor estimado. Baseandose nos experimentos realizados, notamos que os melhores tempos de execução do AHEAD, para diversos grafos, ocorrem para valores de $k$ no intervalo $1 \leq k \leq 2$. Mais especificamente, para esse trabalho, os ajustes de $B_{\text {hdfs }}$ foram feitos de forma a manter o valor de $k$ próximo de 1. Contudo, o estudo dos efeitos da variação de $k$ devem ser feitos de maneira mais aprofundada e em diferentes contextos do uso da plataforma Hadoop.

\section{F. Comparação HEDA e AHEAD}

Ambos os algoritmos foram executados em diversos grafos usando o mesmo valor de $B_{\text {hdfs. }}$ Um resumo das medições de uso de recursos é exibido na Tabela II. A Figura 9a, exibe os valores de uso da memória no processamento do grafo sintético de $50 \mathrm{~K}$ vértices e $800 \mathrm{~K}$
Tabela II

UTILIZAÇÃo DE RECURSos PELOS ALGORITMOS HEDA E AHEAD

\begin{tabular}{ccccc}
\hline Recurso & Grafo & HEDA & AHEAD & Redução \\
\hline \multirow{3}{*}{ Memória (GB) } & Sintético & 11161,6 & 3487,2 & $68,8 \%$ \\
& Enron & 11152,7 & 3714,1 & $66,7 \%$ \\
& Co-autores & 11157,6 & 3075,5 & $72,4 \%$ \\
\hline \multirow{2}{*}{ Discos (GB) } & Sintético & 875,7 & 573,2 & $34,5 \%$ \\
& Enron & 399,4 & 142,4 & $64,3 \%$ \\
& Co-autores & 182,2 & 90,5 & $50,3 \%$ \\
\hline \multirow{2}{*}{ Tempo (Min.) } & Sintético & 147,9 & 102,8 & $30,5 \%$ \\
& Enron & 67,1 & 40,2 & $40,0 \%$ \\
& Co-autores & 46,7 & 24,5 & $47,5 \%$ \\
\hline
\end{tabular}

arestas. O valor fornecido pela plataforma Hadoop é a soma dos bytes usados por todas as tarefas instanciadas durante o Job. O algoritmo AHEAD reduz em 68,8\% o uso de memória em relação ao algoritmo HEDA, quando somados os valores dos Jobs. A Figura 9b mostra o uso do espaço agregado de disco no cluster. Os valores medidos são referentes à quantidade de dados na saída da fase map, que fica armazenada nos discos locais antes da transferência (shuffle) para os reducers. O algoritmo AHEAD reduz em $34,5 \%$ o uso de espaço em disco, considerando-se a soma de dados armazenada em cada Job.

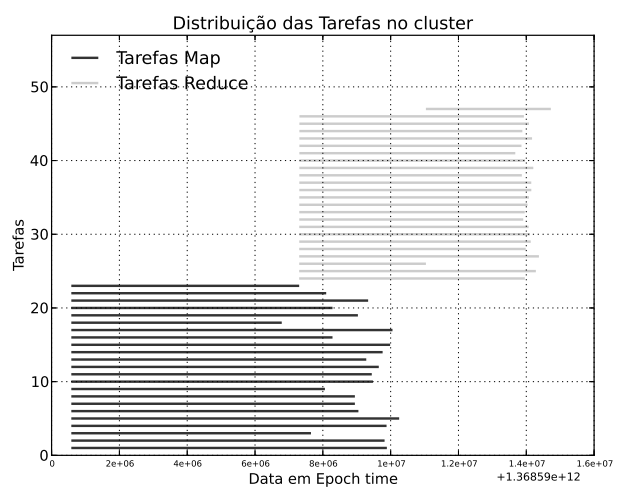

(a) $(\mathrm{k}=1)$

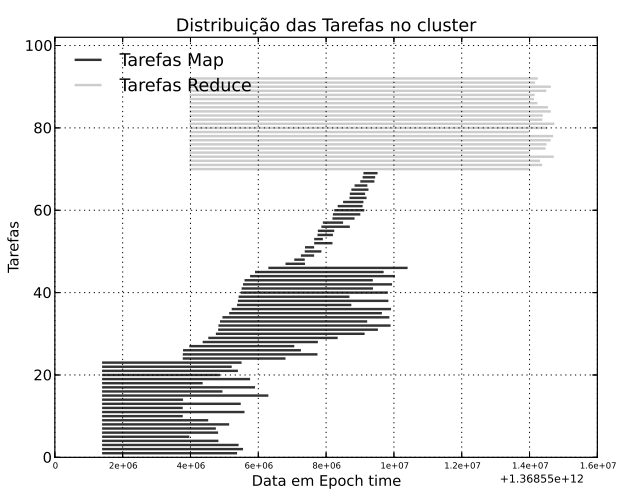

(b) $(\mathrm{k}=2)$

Figura 8. Variação da relação entre o número de mappers e reducers no processamento do grafo Epinions. Para $k=1$ o número de mappers é igual ao número de reducers. Para $k=2$ o número de mappers esperado é o dobro do número de reducers, porém o valor de $T_{e}$ foi um pouco acima do estimado, causando o instanciamento de tarefas map (tarefas 47 a 69) com curto tempo de processamento. 


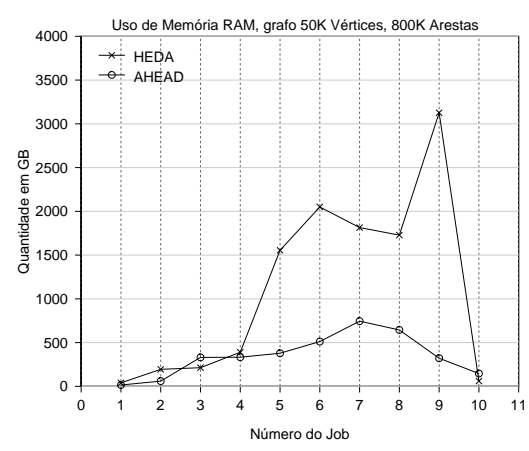

(a) Uso acumulativo da memória RAM no cluster.

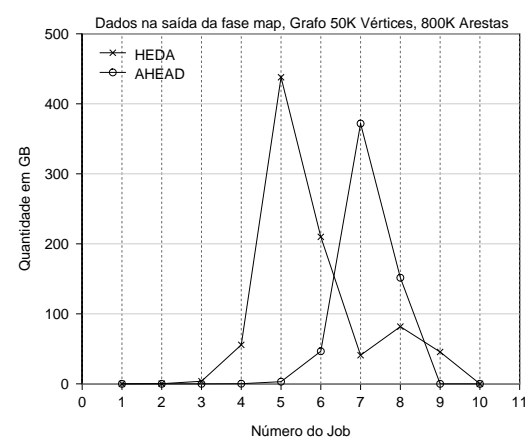

(b) Uso de espaço do sistema de arquivo agregado no cluster.

Figura 9. Comparação do uso do sistema de arquivo agregado e da memória RAM pelos algoritmos HEDA e AHEAD.

\section{Conclus Ão e Trabalhos Futuros}

Esse artigo apresentou o algoritmo paralelo AHEAD, desenvolvido para o processamento eficiente e exato de medidas de centralidades em grafos grandes. Experimentos foram feitos para demonstrar a escalabilidade, o spee$d u p$ e a eficiência do algoritmo. Com a implementação das técnicas In-Mapper Combiner e Schimmy para algoritmos paralelos implementados no modelo MapReduce/Hadoop, foi possível reduzir em até $72,4 \%$ o uso da memória RAM e em até $64,3 \%$ o uso do espaço em disco em relação ao algoritmo HEDA. A principal contribuição desse trabalho é o projeto e a implementação de um algoritmo que possibilita o processamento de grafos maiores, a partir do uso mais eficiente dos recursos computacionais disponíveis.

Trabalhos futuros devem investigar qual é a melhor relação entre o número de mappers e reducers em um cluster Hadoop executando o AHEAD. Além disso, a implementação do AHEAD pode ser aprimorada por meio do particionamento do grafo procurando minimizar o número de vértices vizinhos em partições diferentes e, ao mesmo tempo, manter o tamanho das mesmas relativamente iguais.

\section{AGRADECIMENTOS}

Os autores agradecem ao INCT InWeb, ao CNPQ e à FAPEMIG pelo apoio.

\section{REFERÊNCIAS}

[1] R. Bryant, "Data-intensive scalable computing for scientific applications," Computing in Science \& Engineering, vol. 13, no. 6, pp. 25-33, Nov.-Dec. 2011.
[2] L. C. Freeman, "Centrality in social networks conceptual clarification," Social Networks, vol. 1, no. 3, pp. 215 -239, 1979.

[3] U. Kang, S. Papadimitriou, J. Sun, and H. Tong, "Centralities in large networks: Algorithms and observations," in SIAM International Conference on Data Mining. SIAM / Omnipress, Apr 2011b, pp. 119-130.

[4] U. Kang, C. E. Tsourakakis, A. P. Appel, C. Faloutsos, and J. Leskovec, "Hadi: Mining radii of large graphs," $A C M$ Trans. Knowl. Discov. Data, vol. 5, no. 2, pp. 8:1-8:24, Feb 2011a.

[5] J. P. B. Nascimento and C. D. Murta, "Um Algoritmo Paralelo para Cálculo de Centralidade em Grafos Grandes," in Anais do XXX SBRC, 2012, pp. 393-406.

[6] J. P. B. Nascimento, "Um Algoritmo Paralelo para Cálculo de Centralidade em Grafos Grandes," Master's thesis, PPGMMC, CEFET-MG, 2011.

[7] J. Lin and M. Schatz, "Design patterns for efficient graph algorithms in MapReduce," in Proceedings of the Eighth Workshop on Mining and Learning with Graphs, ser. MLG '10. ACM, 2010, pp. 78-85.

[8] J. Dean and S. Ghemawat, "MapReduce: Simplified data processing on large clusters," in USENIX Association Proceedings of the Sixth Symposium on Operating Systems Design and Implementation (OSDE'04). USENIX ASSOC, 2004, pp. 137-149.

[9] - "Mapreduce: simplified data processing on large clusters," Commun. ACM, vol. 51, no. 1, pp. 107-113, Jan 2008.

[10] H. Karloff, S. Suri, and S. Vassilvitskii, "A model of computation for MapReduce," in Proceedings of the TwentyFirst Annual ACM-SIAM Symposium on Discrete Algorithms, ser. SODA'10. Society for Industrial and Applied Mathematics, 2010, pp. 938-948.

[11] S. N. Srirama, P. Jakovits, and E. Vainikko, "Adapting scientific computing problems to clouds using MapReduce," Future Gener. Comput. Syst., vol. 28, no. 1, pp. 184-192, Jan 2012.

[12] J. Cohen, "Graph Twiddling in a MapReduce World," Computing in Science \& Engineering, vol. 11, no. 4, pp. 29-41, Jul-Aug 2009.

[13] L. Page, S. Brin, R. Motwani, and T. Winograd, "The pagerank citation ranking: Bringing order to the web." Stanford InfoLab, Technical Report 1999-66, November 1999.

[14] P. Flajolet and G. N. Martin, "Probabilistic counting algorithms for data base applications," J. Comput. Syst. Sci., vol. 31, no. 2, pp. 182-209, Sep. 1985.

[15] A. A. Hagberg, D. A. Schult, and P. J. Swart, "Exploring network structure, dynamics, and function using NetworkX," in Proceedings of the 7th Python in Science Conference (SciPy2008), Aug. 2008, pp. 11-15.

[16] M. R. S. Gonçalves, J. N. Maciel, and C. D. Murta, "Geração de Topologias da Internet por Redução do Grafo Original." in Anais do XXVIII SBRC, 2010, pp. 959-972.

[17] L. C. da Cruz, "Um Algoritmo Paralelo Eficiente para Cálculo de Centralidade em Grafos," Master's thesis, PPGMMC, CEFET-MG, 2013. 